\title{
Physics considerations for laser-plasma linear colliders
}

\author{
C. B. Schroeder, E. Esarey, C. G. R. Geddes, C. Benedetti, and W. P. Leemans \\ Lawrence Berkeley National Laboratory, Berkeley, California 94720, USA
}

(Received 11 June 2010; published 4 October 2010)

\begin{abstract}
Physics considerations for a next-generation linear collider based on laser-plasma accelerators are discussed. The ultrahigh accelerating gradient of a laser-plasma accelerator and short laser coupling distance between accelerator stages allows for a compact linac. Two regimes of laser-plasma acceleration are discussed. The highly nonlinear regime has the advantages of higher accelerating fields and uniform focusing forces, whereas the quasilinear regime has the advantage of symmetric accelerating properties for electrons and positrons. Scaling of various accelerator and collider parameters with respect to plasma density and laser wavelength are derived. Reduction of beamstrahlung effects implies the use of ultrashort bunches of moderate charge. The total linac length scales inversely with the square root of the plasma density, whereas the total power scales proportional to the square root of the density. A $1 \mathrm{TeV}$ center-ofmass collider based on stages using a plasma density of $10^{17} \mathrm{~cm}^{-3}$ requires tens of $J$ of laser energy per stage (using $1 \mu \mathrm{m}$ wavelength lasers) with tens of $\mathrm{kHz}$ repetition rate. Coulomb scattering and synchrotron radiation are examined and found not to significantly degrade beam quality. A photon collider based on laser-plasma accelerated beams is also considered. The requirements for the scattering laser energy are comparable to those of a single laser-plasma accelerator stage.
\end{abstract}

DOI: 10.1103/PhysRevSTAB.13.101301

PACS numbers: 52.38.Kd, 41.75.Lx

\section{INTRODUCTION}

Advanced acceleration techniques are actively being pursued to expand the energy frontier of future colliders. Although the minimum energy of interest for the next lepton collider will be determined by high-energy physics experiments that are presently underway, it has long been anticipated that $\geq 1 \mathrm{TeV}$ center-of-mass energy will be required $[1,2]$. This center-of-mass energy is already near the limit of what can be constructed using conventional accelerator technology, given reasonable space and cost restrictions [3].

Laser-plasma accelerators (LPAs) [4] are of great interest because of their ability to sustain extremely large acceleration gradients, enabling compact accelerating structures. Laser-plasma acceleration is realized by using a short-pulse, high-intensity laser to ponderomotively drive a large electron plasma wave (or wakefield) in an underdense plasma. The electron plasma wave has relativistic phase velocity, approximately the group velocity of the laser, and can support large electric fields in the direction of propagation of the laser. When the laser pulse is approximately resonant, with duration on the order of the plasma period, and the laser intensity is relativistic, with normalized laser vector potential $a_{0}=e A / m_{e} c^{2} \sim 1$, the size of the accelerating field is on the order of $E_{0}=$ $c m_{e} \omega_{p} / e$, or $E_{0}[\mathrm{~V} / \mathrm{m}] \simeq 96 \sqrt{n_{0}\left[\mathrm{~cm}^{-3}\right]}$, where $\omega_{p}=$ $c k_{p}=\left(4 \pi n_{0} e^{2} / m_{e}\right)^{1 / 2}=2 \pi c / \lambda_{p}$ is the electron plasma frequency, $n_{0}$ is the ambient electron number density, $m_{e}$ and $e$ are the electron rest mass and charge, respectively, and $c$ is the speed of light in vacuum. For example, fields on the order of $E_{0} \simeq 100 \mathrm{GV} / \mathrm{m}$ are generated at plasma densities of $n_{0}=10^{18} \mathrm{~cm}^{-3}$. LPAs have demonstrated (e.g., Ref. [5]) accelerating gradients on the order of $100 \mathrm{GV} / \mathrm{m}$, several orders of magnitude larger than conventional accelerators, which are presently limited to $\sim 100 \mathrm{MV} / \mathrm{m}$ by material breakdown. Hence, employing LPA technology has the potential to significantly reduce the main linac length (and, therefore, the cost) of a future lepton collider [6,7].

Rapid progress in the field of laser-plasma acceleration, and in particular the demonstration of high-quality $\mathrm{GeV}$ electron beams using $\mathrm{cm}$-scale plasmas at Lawrence Berkeley National Laboratory [8,9], has increased interest in laser-plasma acceleration as a path toward a compact TeV-class linear collider. In this paper, we consider several aspects of the design of a TeV-class linear collider based on LPAs, and discuss several of the advantages and challenges of LPA technology. Here we focus on the main LPA-based linacs. Other collider components, such as the injector (and cooling systems) and beam delivery to the interaction point, are not addressed in this work.

\section{LASER-PLASMA ACCELERATORS}

In this section the physics of laser-plasma acceleration relevant to collider design is reviewed. In the standard configuration, the electron plasma wave is driven by an intense laser pulse with duration on the order of the plasma period propagating in a neutral, underdense plasma $\left(\lambda_{p} \gg\right.$ $\lambda$, where $\lambda$ is the laser wavelength). The ions are immobile on the time scale of the laser-plasma interaction (valid provided $a_{0}^{2} \ll M_{i} / Z m_{e}$, where $M_{i}$ is the ion mass and $Z$ the charge state). The large amplitude plasma waves (fields 
on the order of $E_{0}$ ) have relativistic phase velocities and can accelerate charged particle beams. Given finite laser driver energy, achieving high beam energy requires staging multiple LPAs [6,7].

There are several regimes of plasma acceleration that can be accessed with a laser driver [4]. Two regimes that have attracted attention for collider applications are the quasilinear regime and the bubble [10] (or blowout [11]) regime. The quasilinear regime is accessible for parameters such that $k_{p}^{2} r_{L}^{2} / 2>a_{0}^{2} / \gamma_{\perp}$ and $a_{0}^{2} \sim 1$, where $r_{L}$ is the characteristic scale length of the transverse laser intensity, $\gamma_{\perp}=\left(1+a_{0}^{2} / 2\right)^{1 / 2}$, and $a_{0}^{2}=7.3 \times$ $10^{-19}(\lambda[\mu \mathrm{m}])^{2} I_{0}\left[\mathrm{~W} / \mathrm{cm}^{2}\right]$, with $I_{0}$ the laser intensity, assuming linear polarization [4]. This regime is characterized by regular plasma wave buckets and nearly symmetric regions of acceleration-deceleration and focusingdefocusing [cf. Figs. 1(a)-1(c)]. The amplitude of the

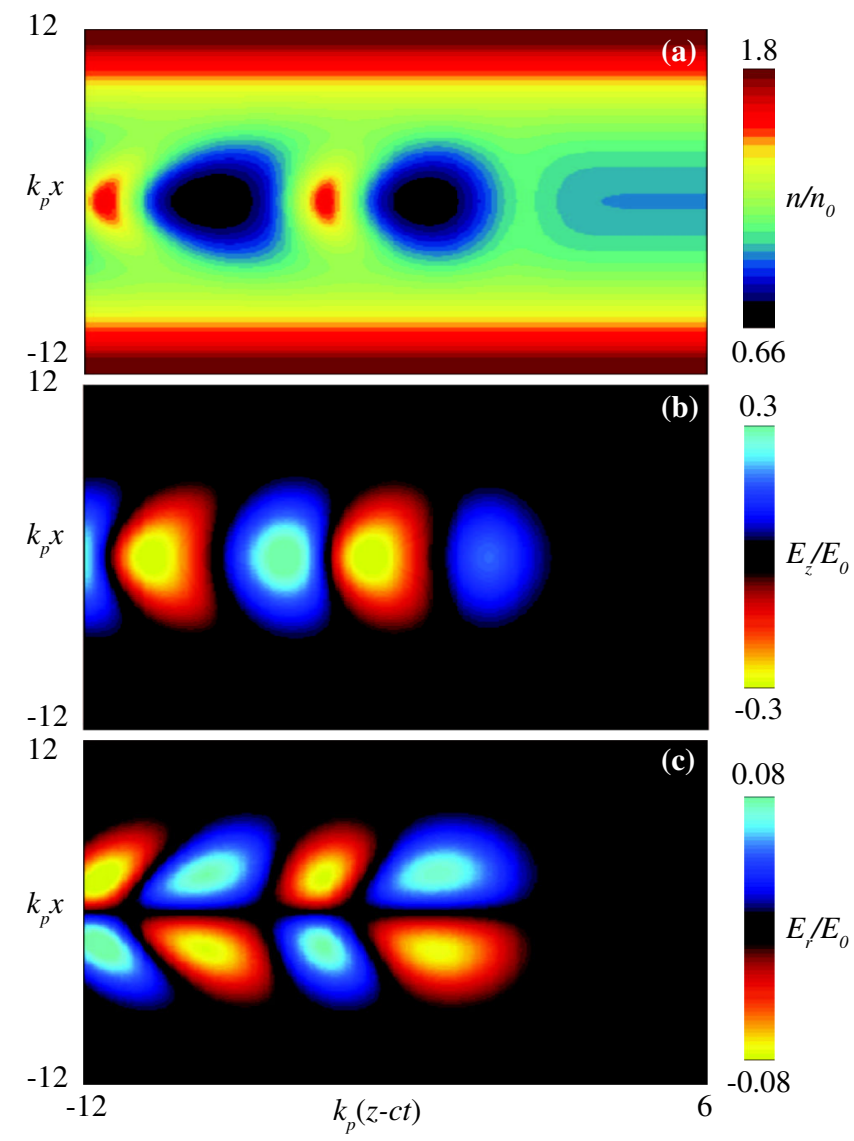

FIG. 1. Plasma wave excitation in the quasilinear regime: (a) electron density $n / n_{0}$, (b) axial electric field $E_{z} / E_{0}$, (c) transverse electric field $E_{r} / E_{0}$. Profile of the normalized laser vector potential initially has the form $a=$ $a_{0} \exp \left(-r^{2} / r_{L}^{2}-z^{2} / 4 L^{2}\right)$, with $a_{0}=1, k_{p} r_{L}=5$, and $k_{p} L=$ 1. Laser is propagating to the right [centered at $k_{p}(z-c t)=0$ ] in a matched parabolic plasma density channel. Accessible phase regions of focusing and acceleration for both electrons and positrons are present. accelerating field of the plasma wave is approximately $E_{z} \approx 0.76\left(a_{0}^{2} / 2 \gamma_{\perp}\right) E_{0}$ for a resonant Gaussian laser pulse. In the quasilinear regime, the accelerating and focusing phase regions for electrons and positrons are nearly symmetric since the wakefield is approximately sinusoidal. Figure 1 includes a plasma channel (parabolic transverse plasma density variation) for laser guiding. As shown in Fig. 1, the plasma channel results in curvature of the plasma wave and a phase shift between the accelerating and focusing phase regions that increases for accelerating buckets farther behind the drive laser [12].

The bubble regime of LPA occurs for laser-plasma parameters such that $k_{p} r_{L} \lesssim 2 \sqrt{a_{0}}$, assuming $a_{0}^{2}>1$ [11]. This regime is characterized by complete removal of plasma electrons and creation of an electron-free cavity [cf. Figs. 2(a)-2(c)]. The bubble regime has several attractive features for acceleration of electron beams. Inside the moving cavity, the focusing forces for electrons are linear

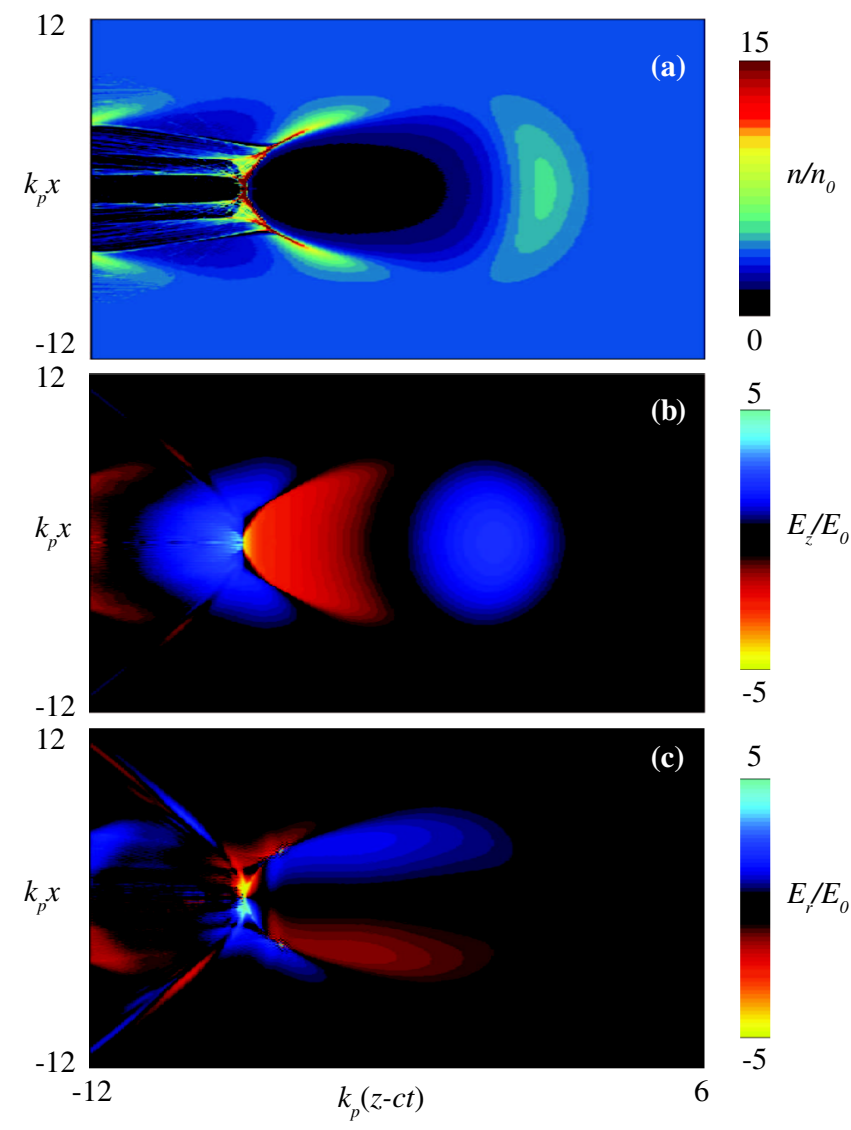

FIG. 2. Plasma wave excitation in the highly nonlinear cavitated regime: (a) electron density $n / n_{0}$, (b) axial electric field $E_{z} / E_{0}$, (c) transverse electric field $E_{r} / E_{0}$. Profile of the normalized laser vector potential initially has the form $a=$ $a_{0} \exp \left(-r^{2} / r_{L}^{2}-z^{2} / 4 L^{2}\right)$, with $a_{0}=4, k_{p} r_{L}=5$, and $k_{p} L=$ 1. Laser is propagating to the right [centered at $k_{p}(z-c t)=0$ ] in initially uniform plasma. Positron acceleration and focusing only possible in electron density spike at the back of the cavity $k_{p}(z-c t) \approx-7.5$. 
(and attractive) and uniform for all phases, and the accelerating field is independent of transverse position with respect to the cavity axis. The major drawback of accessing the highly nonlinear bubble regime is that acceleration of positrons is problematic because the entire cavity is defocusing for positrons, and a positron beam will be scattered transversely. There is a transition regime between the bubble and the quasilinear regimes where the wave is highly nonlinear, but a completely electron-free cavity is not fully formed. In this case there is a small phase region where positrons can be accelerated and focused [cf. Fig. 2 (c)]. (The phase region for positron acceleration in beamdriven plasma wakefield accelerators was analyzed in Refs. $[13,14]$.) This phase region corresponds to the location of the plasma electron density peak and the attractive properties of the bubble regime (e.g., uniform accelerating and constant linear focusing) are lost.

Operating in the bubble regime also limits the flexibility of the accelerator. In the bubble regime the focusing forces and the accelerating forces are determined primarily by the ambient plasma density (background ion density in the cavity). Operating in the quasilinear regime allows independent control of the amplitude of the transverse and longitudinal fields by controlling the shape of the laser profile. The transverse focusing force in the quasilinear regime scales as $F_{\perp} \propto \nabla_{\perp} a^{2}$, and, therefore, by shaping the transverse profile of the laser, the transverse forces in the accelerator can be controlled [15]. Control over the focusing forces enables control of the beam dynamics (e.g., the beam matching condition [16]). Controlling the beam radius is particularly important to avoid deleterious effects, such as beam self-focusing (pinching) and ion motion [17].

In the quasilinear regime (with $a_{0} \sim 1$ ), efficient excitation of the plasma wave requires the laser duration to be on the order of the plasma period such that $\omega_{p} \tau_{L} \sim 1$, where $\tau_{L}$ is the rms duration of the laser intensity. Hence, the required laser pulse duration scales with density as $\tau_{L} \propto n_{0}^{-1 / 2}$. Operating in the quasilinear regime also puts bounds on the laser spot size. To avoid bubble formation requires $k_{p}^{2} r_{L}^{2} / 2>a_{0}^{2} / \gamma_{\perp}$. In addition, to avoid transverse laser intensity evolution (strong self-focusing) the laser power must be less than or on the order of the critical power $P / P_{c}=\left(k_{p} r_{L} a_{0}\right)^{2} / 32 \lesssim 1$. These conditions imply $3 \lesssim k_{p} r_{L} \leqslant 6$. Hence, for fixed $a_{0}$ and $k_{p} r_{L}$, the required peak laser power per LPA stage scales as $P_{L} \propto n_{0}^{-1} \lambda^{-2}$, and the laser energy per LPA stage scales as $U_{L} \sim P_{L} \tau_{L} \propto n_{0}^{-3 / 2} \lambda^{-2}$.

The amount of charge that can be accelerated in a plasma wave is determined by the plasma density and the size of the accelerating field. The maximum charge that can be loaded is given approximately by the number of charged particles required to cancel the laser-excited wakefield (beam loading limit). A collider will operate with asymmetric shaped particle bunches such that bunches can be loaded with charge near the beam loading limit without a large wakefield-induced energy spread $[18,19]$. The maximum number of loaded charged particles into a short $\left(\ll \lambda_{p}\right)$ and narrow $\left(\ll \lambda_{p}\right)$ segment scales as $N \sim$ $\pi n_{0} k_{p}^{-3}\left(E_{z} / E_{0}\right)$. Hence, the number of beam particles that can be accelerated scales with plasma density as $N \propto$ $n_{0}^{-1 / 2}$, assuming fixed $E_{z} / E_{0}$ (i.e., fixed $a_{0}$ and $\omega_{p} \tau_{L}$ ). Lower plasma density allows more charge to be loaded in a plasma wave bucket.

In general, the energy gain in a single LPA stage may be limited by laser diffraction effects, dephasing of the electrons with respect to the accelerating field phase velocity (approximately the drive laser velocity), and laser energy depletion into the plasma wave. Laser diffraction effects can be mitigated by use of a plasma channel (transverse plasma density tailoring), guiding the laser over many Rayleigh ranges $[8,20]$. Dephasing can be mitigated by plasma tapering (longitudinal plasma density tailoring), which can maintain the position of the electron beam at a given phase of the plasma wave [21,22]. Hence, the single-stage energy gain is ultimately determined by laser energy depletion. The energy depletion length [23] scales as $L_{d} \sim \lambda_{p}^{3} / \lambda^{2} \propto n_{0}^{-3 / 2} \lambda^{-2}$, and the energy gain in a single stage scales with plasma density and laser wavelength as $W_{\text {stage }} \propto E_{0} L_{d} \propto n_{0}^{-1} \lambda^{-2}$. The number of stages required to reach a required beam energy scales as $N_{\text {stage }} \propto$ $1 / W_{\text {stage }} \propto n_{0} \lambda^{2}$.

After a single LPA stage, the laser energy is depleted and a new laser pulse must be coupled into the plasma for further acceleration. This coupling distance is critical to determining the overall accelerator length (which sets the average, or geometric, gradient of the main linac) and the optimal plasma density at which to operate. One major advantage of laser-driven plasma acceleration is the potential for a short coupling distance between stages, and, therefore, the possibility of a high-average (geometric) accelerating gradient and a relatively short main linac length.

Although conventional laser optics might require a few meters of space to focus intense lasers into subsequent LPA stages, plasma mirrors show great promise as optics to direct high-intensity laser pulses, requiring only tens of $\mathrm{cm}$ to couple a drive laser into a plasma accelerator stage. A plasma mirror uses overdense plasma creation by the intense laser on a renewable surface (e.g., metallic tape or liquid jet) to reflect the laser beam, and such mirrors are in use as optical elements for temporal contrast improvement of ultrashort laser pulses [24,25]. Preliminary experiments [26] are underway to demonstrate the applicability of plasma mirror technology to LPA staging.

The overall linac length will be given by $L_{\text {total }}=$ $\left[L_{\text {stage }}+L_{c}\right] E_{b} / W_{\text {stage }}$, where $L_{c}$ is the required coupling distance for a new drive laser (and space for any required beam transport and diagnostics), $E_{b}$ is the final beam energy, and $L_{\text {stage }} \approx L_{d}$ is the single-stage plasma length 


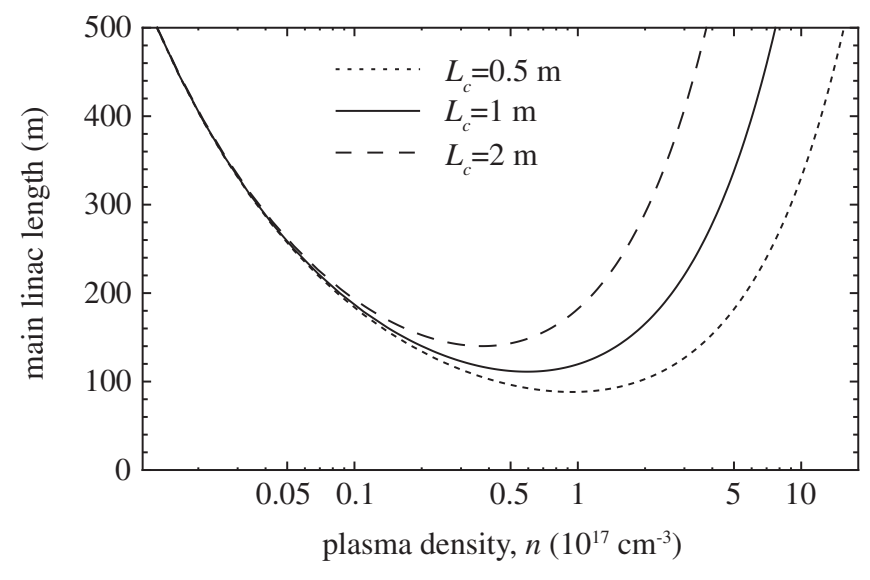

FIG. 3. Main single-linac length versus plasma density $n$ for several laser in-coupling distances $L_{c}$, for $E_{b}=0.5 \mathrm{TeV}$ and $a_{0}=1.5$.

(approximately the depletion length). Figure 3 plots the main linac length versus plasma density for several coupling distances, with $E_{b}=0.5 \mathrm{TeV}$ and $a_{0}=1.5$. Here the single-stage length and energy gain were calculated using a fluid code [27] to model the laser-plasma interaction. TeV beam energies can be obtained in a few hundred meters using staged LPAs. Minimization of the main linac length for coupling distances $\lesssim 1 \mathrm{~m}$ requires operation at densities on the order of $10^{17} \mathrm{~cm}^{-3}$. Reducing the main linac length requires $L_{c} \sim L_{\text {stage }} \approx L_{d}$. Therefore, the length of the main linac scales with density as $L_{\text {total }} \propto L_{d} N_{\text {stage }} \propto$ $n_{0}^{-1 / 2}$. Although operating at a low plasma density increases the single-stage energy gain, it also reduces the accelerating gradient and increases the laser depletion length, thereby increasing the overall linac length.

\section{LASER-PLASMA LINEAR COLLIDER DESIGN CONSIDERATIONS}

The rate of events in a collider is determined by the product of the collision cross section and luminosity. The geometric luminosity is

$$
\mathcal{L}=\frac{f N^{2}}{4 \pi \sigma_{x} \sigma_{y}}=\frac{P_{b}}{4 \pi E_{b}} \frac{N}{\sigma_{x} \sigma_{y}},
$$

where $f$ is the collision frequency, $N$ is the number of particles per bunch (equal number of particles per bunch in the electron and positron beams is assumed), $\sigma_{x}$ and $\sigma_{y}$ are the horizontal and vertical rms beam sizes, respectively, at the interaction point (IP), $E_{\mathrm{cm}}=2 \gamma m c^{2}=2 E_{b}$ is the center-of-mass energy, and $P_{b}=f N E_{b}$ is power in one beam. Since the cross section for collisions scales as the inverse of the square of the center-of-mass energy, $\propto E_{\mathrm{cm}}^{-2}$, the luminosity must increase proportionally to maintain the collision rate. The luminosity requirement is approximately $\mathcal{L}\left[10^{34} \mathrm{~cm}^{-2} \mathrm{~s}^{-1}\right] \approx E_{\mathrm{cm}}^{2}[\mathrm{TeV}]$. As the luminosity scaling indicates, for fixed beam power, the transverse beam density at the IP $\left(N / \sigma_{x} \sigma_{y}\right)$ must be increased as the center-of-mass energy increases. In addition to an electron-positron collider, in Appendix $\mathrm{C}$ we consider the option of a gamma-gamma collider [28,29], where the photon beam luminosity will be reduced from the above geometric luminosity due to the conversion efficiency of electrons to gamma rays.

There are several limitations to the achievable beam density at the IP. Some of these include, for example, the achievable beam emittance (given limitations on initial emittance and cooling methods), radiation effects during the final focus to the IP, emittance growth in the main linacs, and beam-beam interactions at the collision. As discussed below, the beam-beam interaction at the IP implies the need for ultrashort bunches. Ultrashort bunches are intrinsically generated using plasma-based accelerators. This allows suppression of radiation generated by the beam-beam interaction. An emittance growth mechanism unique to plasma-based accelerators is emittance growth due to Coulombic scattering of the beam with background plasma ions. The emittance growth due to the background plasma ions is examined in Appendix A. Beam quality degradation due to synchrotron radiation emission via the strong focusing forces of the plasma wave is addressed in Appendix B. Both Coulomb scattering and synchrotron radiation emission are shown to result in acceptable beam quality degradation. In addition, there are many other sources of beam quality reduction in the linac that are present in any collider design, e.g., misalignment between stages and fluctuations in the accelerator parameters, that are not addressed in this work.

\section{A. Beam-beam interaction}

The beam-beam interaction at the IP produces radiation (beamstrahlung) that generates background for the detectors and increases the beam energy spread (resulting in loss of measurement precision). The beam-beam interaction is characterized by the Lorentz-invariant beamstrahlung parameter (mean field strength in the beam rest frame normalized to the Schwinger critical field) [30]:

$$
\begin{aligned}
\Upsilon & \simeq \frac{5 r_{e}^{2} \gamma N}{6 \alpha \sigma_{z}\left(\sigma_{x}+\sigma_{y}\right)} \\
& =\frac{5 \sqrt{\pi} r_{e}^{2}}{3 \alpha m c^{2}}\left(\frac{E_{b}^{3} \mathcal{L}}{P_{b}}\right)^{1 / 2}\left[\frac{\sqrt{\left(\sigma_{x} / \sigma_{y}\right)}}{1+\left(\sigma_{x} / \sigma_{y}\right)}\right] \frac{N^{1 / 2}}{\sigma_{z}},
\end{aligned}
$$

where $r_{e}=e^{2} / m c^{2}$ is the classical electron radius, $\alpha=$ $e^{2} \hbar / c$ is the fine structure constant, $\sigma_{z}$ is the bunch length, and $\sigma_{x} / \sigma_{y}$ is the aspect ratio of the beam at IP. As Eq. (2) indicates, using flat beams $\sigma_{x} / \sigma_{y} \ll 1$ reduces the beamstrahlung. Round beams may also be considered, as round beams potentially remove the need for damping rings (to produce asymmetric emittances with large aspect ratios), and reduce deleterious wakefield effects. In terms of the 
beamstrahlung parameter, the average number of emitted photons per electron is $n_{\gamma} \simeq 2.54\left(\alpha^{2} \sigma_{z} / r_{e} \gamma\right) \Upsilon(1+$ $\left.\Upsilon^{2 / 3}\right)^{-1 / 2}$ and the relative energy spread induced is $\delta_{E} \simeq$ $1.24\left(\alpha^{2} \sigma_{z} / r_{e} \gamma\right) \Upsilon^{2}\left[1+(3 \Upsilon / 2)^{2 / 3}\right]^{-2}[30]$.

The present generation of linear collider designs based on conventional technology operate in the classical beamstrahlung regime $Y \ll 1$. The next-generation linear colliders with $E_{\mathrm{cm}} \gtrsim 1 \mathrm{TeV}$ will most likely operate in the quantum beamstrahlung regime with $Y \gg 1$. In the quantum beamstrahlung regime $Y \gg 1, n_{\gamma} \propto \sigma_{z} Y^{2 / 3}$ and $\delta_{E} \propto \sigma_{z} \Upsilon^{2 / 3}$. Assuming the collider parameters $E_{\mathrm{cm}}$ and $\mathcal{L}$, and the IP focusing $\sigma_{x}$ and $\sigma_{y}$, are fixed, the number of beamstrahlung photons scale as $n_{\gamma} \propto N^{2 / 3} \sigma_{z}^{1 / 3}$ and the induced beam energy spread scales as $\delta_{E} \propto N^{2 / 3} \sigma_{z}^{1 / 3}$ in the regime $Y \gg 1$. If the collider parameters $E_{\mathrm{cm}}, \mathcal{L}$, and $\sigma_{x} / \sigma_{y}$ are fixed, with a fixed available beam power $P_{b}$, then, in the $Y \gg 1$ regime, the number of beamstrahlung photons scale as $n_{\gamma} \propto\left(N \sigma_{z}\right)^{1 / 3}$ and the induced beam energy spread scales as $\delta_{E} \propto\left(N \sigma_{z}\right)^{1 / 3}$. From these scalings, in the $Y \gg 1$ regime, beamstrahlung is reduced by using shorter bunches and smaller charge per bunch. Reduction in charge per bunch is clearly limited by luminosity requirements (i.e., if the bunch number decreases, then $f$ must be increased or the beam transverse dimen-
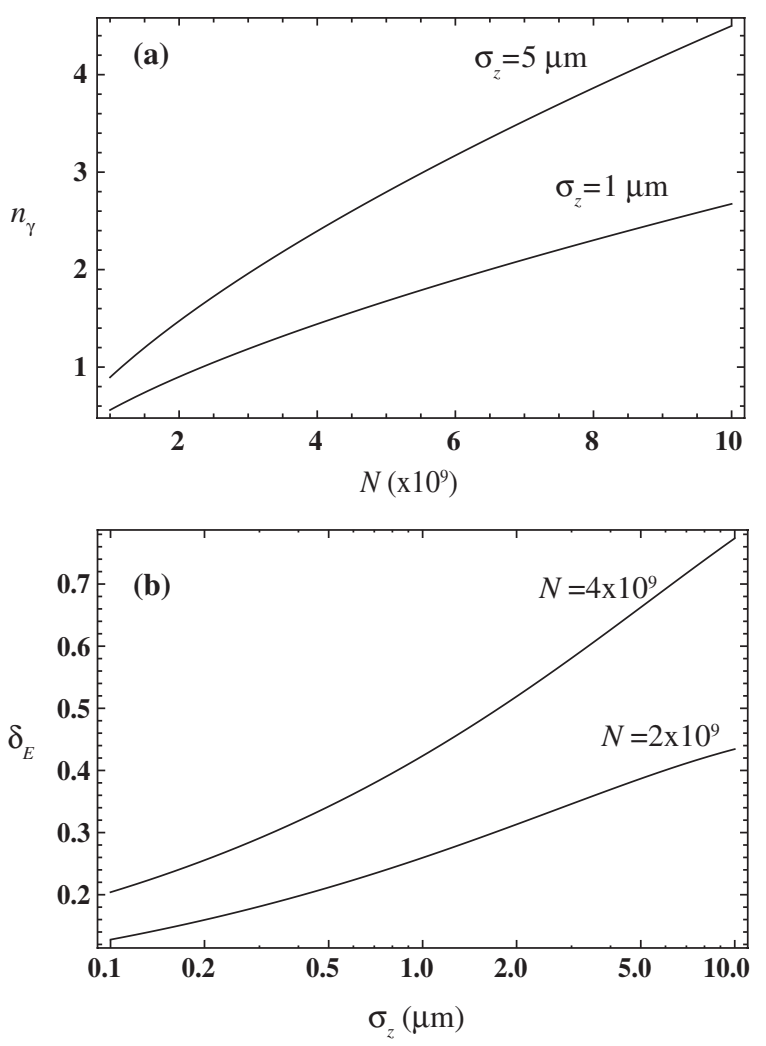

FIG. 4. (a) Beamstrahlung photons emitted per electron $n_{\gamma}$ versus $N$ for bunch lengths $\sigma_{z}=1 \mu \mathrm{m}$ and $5 \mu \mathrm{m}$. (b) Beamstrahlung induced energy spread $\delta_{E}$ versus bunch length $\sigma_{z}$ for $N=2 \times 10^{9}$ and $N=4 \times 10^{9}$. sions decreased). For fixed beamstrahlung $n_{\gamma}$ and $\delta_{E}$ (that is acceptable given the collider detector), the luminosity per unit beam power scales as $\mathcal{L} / P_{b} \propto \sigma_{z}^{-1 / 2}$, indicating short bunches are critical for next-generation linear colliders [3].

Figure 4(a) shows $n_{\gamma}$ versus number per bunch $N$ for bunch lengths $\sigma_{z}=1 \mu \mathrm{m}$ and $5 \mu \mathrm{m}$, and Fig. 4(b) shows $\delta_{E}$ versus bunch length $\sigma_{z}$ for $N=2 \times 10^{9}$ and $N=4 \times 10^{9}$. Unless otherwise noted the parameters of Table I were assumed. For a $1 \mathrm{TeV}$ collider, micron bunch lengths are desirable using bunches with a few $10^{9}$ particles. Note that here we have considered round beams. As Eq. (2) indicates, using flat beams will reduce the beamstrahlung: $n_{\gamma} \propto\left(\sigma_{x} / \sigma_{y}\right)^{1 / 3}$ and $\delta_{E} \propto\left(\sigma_{x} / \sigma_{y}\right)^{1 / 3}$ for $Y \gg 1$.

Plasma-based accelerators are intrinsically sources of ultrashort bunches since the scale length of the accelerating bucket in a plasma-based accelerator is the plasma wavelength, i.e., $\sigma_{z} \ll \lambda_{p}$, where the plasma wavelength is $\lambda_{p}[\mu \mathrm{m}] \simeq 33 / \sqrt{n\left[10^{18} \mathrm{~cm}^{-3}\right]}$. In principle, controlled injection in the plasma could achieve high beam quality (low emittance) and ultrashort durations beyond state-ofthe-art photocathodes, due to the space-charge shielding provided by the ions in the plasma and the rapid acceleration facilitated by the ultrahigh accelerating gradients

TABLE I. Example parameters for a $1 \mathrm{TeV}$ laser-plasma linear collider.

\begin{tabular}{lc}
\hline \hline Plasma number density, $n_{0}\left[\mathrm{~cm}^{-3}\right]$ & $10^{17}$ \\
Energy, center of mass, $E_{\mathrm{cm}}[\mathrm{TeV}]$ & 1 \\
Beam energy, $\gamma m c^{2}[\mathrm{TeV}]$ & 0.5 \\
Luminosity, $\mathcal{L}\left[10^{34} \mathrm{~s}^{-1} \mathrm{~cm}^{-2}\right]$ & 2 \\
Number per bunch, $N\left[10^{9}\right]$ & 4 \\
Collision frequency, $f[\mathrm{kHz}]$ & 15 \\
Beam power, $P_{b}[\mathrm{MW}]$ & 4.8 \\
Bunch length, $\sigma_{z}[\mu \mathrm{m}]$ & 1 \\
Horizontal rms beam size at IP, $\sigma_{x}[\mathrm{~nm}]$ & 10 \\
Vertical rms beam size at IP, $\sigma_{y}[\mathrm{~nm}]$ & 10 \\
Beamstrahlung parameter, $Y$ & 180 \\
Beamstrahlung photons per electron $n_{\gamma}$ & 1.4 \\
Beamstrahlung induced energy spread $\delta_{E}$ & 0.42 \\
Plasma wavelength, $\lambda_{p}[\mu \mathrm{m}]$ & 105 \\
Energy gain per stage, $W_{\text {stage }}[\mathrm{GeV}]$ & 10 \\
Stage length $\left(\mathrm{LPA}+\right.$ laser in-coupling), $L_{\text {stage }}[\mathrm{m}]$ & 2 \\
Laser energy per stage, $U_{L}[\mathrm{~J}]$ & 32 \\
Laser wavelength, $\lambda[\mu \mathrm{m}]$ & 1 \\
Laser pulse duration, $\tau_{L}[\mathrm{fs}]$ & 56 \\
Laser radius, $r_{L}[\mu \mathrm{m}]$ & 70 \\
Initial normalized laser intensity, $a_{0}$ & 1.5 \\
Average laser power per stage $P_{\text {avg }}[\mathrm{kW}]$ & 480 \\
Number of stages $\left(1\right.$ linac), $N_{\text {stage }}$ & 50 \\
Linac length $\left(1\right.$ beam), $L_{\text {total }}[\mathrm{km}]$ & 0.1 \\
Efficiency $($ wall-plug to beam) $[\%]$ & 60 \\
Total wall-plug power, $P_{\text {wall }}[\mathrm{MW}]$ & \\
\hline \hline
\end{tabular}


of the plasma wave. Several methods of controlled electron beam injection are actively being pursued to provide detailed control over the amount of trapped charge and the initial phase-space characteristics. Laser-triggered methods [31,32] and plasma density tailoring [33,34] have been proposed for controlled injection. Triggered injection into accelerating plasma waves via colliding laser pulses has been demonstrated experimentally [35]. Production of electron beams via plasma wave excitation on a negative density gradient has also been achieved [36]. Further development is required to achieve the necessary emittance for collider applications.

Positron beam generation would require a two-stage process similar to conventional collider designs. The first stage would be an LPA producing a $10-100 \mathrm{GeV}$ electron beam in several meters, which would be used to generate gamma rays (via Thomson backscattering or bremsstrahlung in a high- $Z$ target). The gamma rays would interact with a solid target creating a positron beam via photopair production. Cooling of the positron beam would be required. A linear cooling system may be considered with application of the ultrahigh accelerating gradients in LPAs [37]. The need for a positron beam may be removed by considering a gamma-gamma or gamma-electron collider, which can access many of the lepton interactions available in an electron-positron collider [29]. Appendix C discusses the design issues with respect to a gamma-gamma collider.

\section{B. Power considerations}

Operational cost of future linear colliders limit the wallplug power to a few hundred MW. For a constant required luminosity (fixed $E_{b}$ and IP beam focusing), the collision frequency scales with plasma density as $f \propto N^{-2} \propto n_{0}$ (from the scaling on bunch number imposed by beam loading), and the beam power scales as $P_{b}=f N E_{b} \propto$ $n_{0}^{1 / 2}$. The average laser power per stage scales as $P_{\text {avg }}=$ $f U_{L} \propto n_{0}^{-1 / 2} \lambda^{-2}$ and the total wall plug power scales as $P_{\text {wall }} \propto N_{\text {stage }} P_{\text {avg }} \propto n_{0}^{1 / 2}$. Hence, going to lower plasma density reduces the total power requirements, but increases the laser energy per stage and total length of the main linac.

Typical conversion efficiencies are projected to be $\sim 50 \%$ for laser to plasma wave (at $a_{0} \sim 1$ ) [23] and $\sim 40 \%$ for plasma wave to beam (shaped electron beams are assumed to avoid energy spread growth $[18,19])$, such that the overall efficiency from laser to beam is $\sim 20 \%$. Since the laser is only $\sim 50 \%$ depleted at the exit of a stage, some of this laser energy may be recovered. In addition, a trailing antiresonant laser pulse [38-40] (or, alternatively, a properly phased low energy electron beam) could be employed to absorb the energy in the plasma wave and transport the energy out of the plasma to be recovered. If we assume a wall-plug to laser efficiency of $\sim 30 \%$, then the efficiency from wall plug to beam is $\sim 6 \%$. Laser efficiencies of tens of percent are presently beyond state-of-the-art technology for short-pulse, high-intensity lasers. Development of high-efficiency, high average power laser systems using diode-pumped ceramics show promise for greatly increasing the efficiency and average power of short-pulse laser systems [41].

The energy deposited into a single plasma accelerator stage and remaining after passage of the particle beam is an issue. For example, for $n_{0}=10^{17} \mathrm{~cm}^{-3}$ (cf. Table I), about $\sim 10 \mathrm{~J}$ of energy remains in the plasma wave after the beam exits a stage, corresponding to $\sim 150 \mathrm{~kW}$ of power. This is a significant cooling challenge. The time between bunches at $15 \mathrm{kHz}$ is $\sim 67 \mu \mathrm{s}$. Presently, the plasma is produced by a hydrogen discharge capillary (constructed of $\mathrm{Al}_{2} \mathrm{O}_{3}$ ) [42]. This is sufficient time to allow for collisional heating of the capillary walls containing the plasma and recombination of the hydrogen, both of which occur on the $\sim$ ns time scale. Using a H-discharge capillary for the plasma channel creation allows the $\mathrm{H}$ gas to be evacuated and new gas pumped in before the arrival of the next laser, aiding in the plasma cooling. In addition, $\mathrm{Al}_{2} \mathrm{O}_{3}$ has excellent heat conduction properties. As discussed above, the energy remaining in the plasma wave may also be removed by a using a trailing antiresonant laser pulse [38-40] (or properly phased low energy electron beam).

\section{PLASMA DENSITY AND LASER WAVELENGTH SCALINGS}

Table I shows a $1 \mathrm{TeV}$ collider example using $n_{0}=$ $10^{17} \mathrm{~cm}^{-3}$ and $\lambda=1 \mu \mathrm{m}$. Many of the important parameters for collider design scale with the plasma density and laser wavelength. With the scalings known, a single design may be scaled to explore parameter space, given additional constraints. For fixed collider parameters (center-of-mass energy, luminosity, and IP focusing) and fixed normalized LPA parameters $\left(a_{0}, k_{p} r_{L}\right.$, and $\left.\omega_{p} \tau_{L}\right)$, the basic scalings with plasma density and laser wavelength are

$$
\begin{gathered}
E_{z} \propto n_{0}^{1 / 2}, \\
L_{\text {stage }} \propto n_{0}^{-3 / 2} \lambda^{-2}, \\
W_{\text {stage }} \propto n_{0}^{-1} \lambda^{-2}, \\
N_{\text {stage }} \propto n_{0} \lambda^{2}, \\
L_{\text {total }} \propto n_{0}^{-1 / 2}, \\
N \propto n_{0}^{-1 / 2}, \\
\tau_{L} \propto n_{0}^{-1 / 2}, \\
P_{\text {peak }} \propto n_{0}^{-1} \lambda^{-2},
\end{gathered}
$$




$$
\begin{gathered}
U_{L} \propto n_{0}^{-3 / 2} \lambda^{-2}, \\
f \propto n_{0}, \\
P_{b} \propto n_{0}^{1 / 2}, \\
Y \propto n_{0}^{-1 / 2}, \\
P_{\text {avg }} \propto n_{0}^{-1 / 2} \lambda^{-2}, \\
P_{\text {wall }} \propto n_{0}^{1 / 2} .
\end{gathered}
$$

Equations (7) and (16) indicate that the total length of the linac $L_{\text {total }}$ and the required power $P_{\text {wall }}$ are independent of laser wavelength. Note that the bandwidth and wavelength of the laser are determined by the amplifying media. Bandwidth constraints will limit the achievable laser pulse duration and, hence, the plasma density required for a resonant laser pulse $\omega_{p} \tau_{L} \sim 1$. In the quantum beamstrahlung regime $Y \gg 1$, for fixed collider parameters (centerof-mass energy, luminosity, and IP focusing), the number of beamstrahlung photons and induced energy spread scale as $n_{\gamma} \propto \delta_{E} \propto n_{0}^{-1 / 3}$. Note that, in an LPA, the bunch length $\sigma_{z}$ is independent of plasma density (provided $\sigma_{z} \ll \lambda_{p}$ ) and is determined by the trapping physics in the plasmabased injection process. The bunch radius can also be controlled independent of density by varying the laser transverse intensity profile (i.e., focusing forces). In Table I, the electron energy gain per stage and the length of the plasma channel were extrapolated from fluid simulations and assume a mild density taper.

With the plasma density scalings [Eqs. (3)-(16)], the example in Table I can be scaled assuming various constraints. For example, if laser technology dictates that $\tau_{L} \geq$ $250 \mathrm{fs}$ (due to bandwidth constraints of the amplifying media used to generate the high-average power laser), then a plasma density of $n_{0}=5 \times 10^{15} \mathrm{~cm}^{-3}$ should be used for resonance with the plasma period (excitation of large amplitude plasma waves in the quasilinear regime). At this density the laser, plasma, and collider parameters would scale according to Eqs. (3)-(16).

\section{DISCUSSION AND CONCLUSIONS}

In this paper we have discussed several physics considerations for future linear colliders using laser-plasma accelerators. The scaling of various collider and accelerator parameters with respect to plasma density, laser wavelength, and electron bunch length were determined. For example, the total linac length scales as $L_{\text {total }} \propto n_{0}^{-1 / 2}$, whereas the total wall power scales as $P_{\text {wall }} \propto n_{0}^{1 / 2}$. To maintain a high geometric gradient, the length of a single accelerating stage should be on the order of the laser in-coupling length. Assuming a coupling distance of $L_{c} \sim 1 \mathrm{~m}$ implies an operational plasma density on the order of $10^{17} \mathrm{~cm}^{-3}$ using $1 \mu \mathrm{m}$ lasers. Beamstrahlung scalings, e.g., $n_{\gamma} \propto \sigma_{z}^{1 / 3}$ for $Y \gg 1$, favor the use of ultrashort bunches with low charge. A key benefit of LPAs is their ability to generate and accelerate ultrashort (fs) bunches. Based on these considerations, one possible example (with plasma density $n_{0}=10^{17} \mathrm{~cm}^{-3}$ and $1 \mu \mathrm{m}$ laser wavelength) of self-consistent LPA-based collider parameters for $1 \mathrm{TeV}$ center-of-mass energy is presented in Table I. For fixed collider parameters (center-of-mass energy, luminosity, and IP focusing) and fixed normalized LPA parameters $\left(a_{0}, k_{p} r_{L}\right.$, and $\left.\omega_{p} \tau_{L}\right)$, the basic plasma density and laser wavelength scalings [Eqs. (3)-(16)] allow straightforward exploration of parameter space for collider design.

Beam quality degradation mechanisms unique to plasma-based accelerators, namely emittance growth owing to Coulomb scattering with background plasma ions and energy spread growth from synchrotron radiation in the focusing fields of the plasma wave, are considered in Appendices A and B. Both these effects are shown to result in acceptable beam quality degradation. In addition to Coulomb scattering, there are many other sources of emittance dilution in the linac, such as misalignment between accelerating stages, vibrations, and fluctuations in the plasma and laser parameters. In general, the strong focusing of the plasma accelerator results in more stringent alignment tolerances due to the small matched beam spot size. Beyond state-of-the-art beam based alignment techniques would be required to satisfy the alignment tolerances [3].

Several components of an electron-positron collider have not been addressed in this paper, including positron beam creation and cooling, and final focusing. As an alternative to an electron-positron collider, a gammagamma collider driven by laser-plasma acceleration of electron beams may also be considered. This would also eliminate the need for positron creation and, potentially, damping rings. The scattering laser energy and power requirements for the gamma-gamma collider (e.g., few $\mathrm{J}$ of laser energy at the accelerator repetition rate for $E_{b}=$ $0.25 \mathrm{TeV}$ using a $1 \mu \mathrm{m}$ laser wavelength) are similar to those required for the single-stage plasma wave excitation (e.g., tens of $\mathrm{J}$ at the accelerator repetition rate operating at $n_{0}=10^{17} \mathrm{~cm}^{-3}$ and $1 \mu \mathrm{m}$ laser wavelength). Hence, development of laser technology for LPAs will also enable a gamma-gamma collider. Note that the required wavelength of the scattering laser for a gamma production is determined by the beam energy $\lambda_{L}[\mu \mathrm{m}] \approx 4 E_{b}$ [TeV] [28], and higher collider energies require longer laser wavelengths. Higher collider energies also require higher scattering laser energies [cf. Eq. (C2)]. The details of the design considerations for a gamma-gamma collider are discussed in Appendix C. 
Significant laser technology advances are required to realize an LPA linear collider, which, for $n_{0}=$ $10^{17} \mathrm{~cm}^{-3}$, requires tens of $\mathrm{J}$ of laser energy per pulse (using a $1 \mu \mathrm{m}$ laser wavelength) at $\sim 10 \mathrm{kHz}$ repetition rates with high efficiency $(>10 \%)$. Although $\sim 10 \mathrm{~J}$, short-pulse lasers are currently available, repetition rates of $\sim 10 \mathrm{kHz}$ and tens of percent efficiencies are presently beyond state-of-the-art laser technology. Diode-pumped solid-state lasers show promise to generate hundreds of $\mathrm{kW}$ with high efficiency in the next decade. In addition there is significant LPA research and development required before realization of an LPA-based linear collider is possible. In particular, these include demonstration of accelerator stage coupling, detailed control of beam injection, and maintaining high beam quality over the length of the accelerator (i.e., multiple stages). Technologies for controlling the plasma density profile and laser in-coupling techniques are also required. A TeV linear collider is extremely challenging for any technology, but laser-plasmabased accelerators continue to show promise as a potential solution to address the size of future linear colliders.

\section{ACKNOWLEDGMENTS}

The authors would like to thank Csaba Tóth for many valuable discussions on the topic of laser technology. The authors would also like to thank G. Dugan for many useful and enlightening conversations about collider physics. One of the authors (E. E.) also acknowledges useful conversations with R. Aßmann, W. Chou, T. Raubenheimer, M. Seidel, T. Tajima, and K. Yokoya at the Workshop for High Power Laser Technology for Future Accelerators, especially concerning the use of round beams at the IP. This work was supported by the Director, Office of Science, Office of High Energy Physics, of the U.S. Department of Energy under Contract No. DE-AC0205CH11231.

\section{APPENDIX A: EMITTANCE GROWTH VIA PLASMA SCATTERING}

In this Appendix, emittance growth via plasma scattering for laser-driven wakefields in the quasilinear regime is discussed. Emittance growth due to scattering of electrons with ions in the particle beam-driven blowout regime was considered in Ref. [43].

Emittance growth can occur by elastic scattering of the beam and the ions in the plasma. Coulomb collisions between a beam electron and a background ion in the plasma results in a change of the rms divergence of the particle beam [44],

$$
\frac{d\left\langle\theta^{2}\right\rangle}{d z}=\frac{8 \pi n_{i} Z^{2} r_{e}^{2}}{\gamma^{2}} \ln \left(\frac{b_{\max }}{b_{\min }}\right)=\frac{2 k_{p}^{2} r_{e} Z}{\gamma^{2}} \ln \left(\frac{\lambda_{\mathrm{D}}}{R}\right),
$$

where $n_{i}=n_{0} / Z$ is the ion density and $Z$ is the charge state of the ion. Here $b_{\max }=\lambda_{\mathrm{D}}$ is the plasma Debye length (screening is provided by background electrons in the quasilinear regime), and $b_{\min }=R$ is the effective Coulombic radius of the nucleus, which can be approximated as $R \approx 1.4 A^{1 / 3}$ fm [45] with $A$ the mass number.

Assuming linear focusing forces $\left(F_{\perp} / \gamma m c^{2}=-k_{\beta}^{2} x_{\perp}\right)$ and an approximately matched beam, the resulting rms normalized emittance growth is $d \epsilon_{n} / d z=$ $\gamma k_{\beta}^{-1}\langle\theta d \theta / d z\rangle$, or

$$
\frac{d \epsilon_{n}}{d \gamma}=\frac{k_{p}^{2} r_{e} Z \ln \left(\lambda_{\mathrm{D}} / R\right)}{\gamma k_{\beta}(d \gamma / d z)}
$$

Equation (A2) indicates that the strong focusing in a plasma-based accelerator $k_{\beta} \sim k_{p} / \sqrt{\gamma}$ suppresses the emittance growth from scattering. For linear acceleration, the total emittance growth over the length of the accelerator is approximately

$$
\Delta \epsilon_{n} \approx Z r_{e} \Phi \ln \left(\lambda_{\mathrm{D}} / R\right)\left(\gamma_{f}^{1 / 2}-\gamma_{i}^{1 / 2}\right),
$$

where $\gamma_{f}\left(\gamma_{i}\right)$ is the final (initial) beam energy, $\Phi=$ $\left(k_{p} r_{L} / 2\right)\left(E_{z} / E_{0}\right)^{-3 / 2} / \sqrt{\cos ^{2} \Psi \sin \Psi}$, with $\Psi$ the phase of the beam in the quasilinear wakefield, and $r_{L}$ is the characteristic length of the transverse laser intensity gradient. For typical parameters, $\Phi \sim 1$. Note that Eq. (A3) differs from the result of Ref. [43] in the form of the accelerating and focusing fields in the quasilinear regime and in the impact parameters (e.g., screening provided by plasma electrons). Equations (A2) and (A3) indicate that the emittance growth is only weakly dependent on plasma density. Assuming a fully ionized hydrogen plasma with $n_{0}=10^{17} \mathrm{~cm}^{-3}$ and a temperature of $T=10 \mathrm{eV}$ (i.e., $\lambda_{\mathrm{D}}=74 \mathrm{~nm}$ ), a resonant laser pulse with $a_{0}=1.5$ (i.e., $\left.E_{z} / E_{0} \simeq 0.6\right)$ and $k_{p} r_{L}=4$, and a beam injected at $\Psi=10^{\circ}$, the emittance growth via scattering is $\Delta \epsilon_{n} \approx$ $0.5 \mathrm{~nm} \mathrm{rad}$ after acceleration to $E_{b}=0.5 \mathrm{TeV}$. For round beam operation at the IP with $E_{\mathrm{cm}}=1 \mathrm{TeV}$, this emittance growth is negligible. For flat beam operation at the IP and higher center-of-mass energies (e.g., $E_{\mathrm{cm}}=10 \mathrm{TeV}$ ), the emittance growth via scattering with the ions can be on the order of the required beam emittance.

\section{APPENDIX B: ENERGY SPREAD GROWTH VIA SYNCHROTRON RADIATION}

A beam in the strong transverse focusing forces of the plasma wave will emit synchrotron radiation. The power radiated via synchrotron radiation [45] is

$$
P=\frac{2 e^{2} \gamma^{2}}{3 m^{2} c^{3}} F_{\perp}^{2}
$$

Consider a linear, with respect to transverse position, transverse force from the plasma wave, $F_{\perp}=$ $-m c^{2} \kappa^{2} x_{\perp}$, where $\kappa$ is a constant determined by the laser-plasma parameters: $\kappa^{2}=\left(4 / r_{L}^{2}\right)\left(E_{z} / E_{0}\right) \sin \Psi$ in the quasilinear regime. The betatron wave number is 
$k_{\beta}=\kappa / \gamma^{1 / 2}$. For a relativistic $(\beta \simeq 1)$ particle, the energy loss via synchrotron radiation is

$$
\frac{d \gamma}{d z}=-\frac{2}{3} r_{e} \gamma^{2}\left(\frac{F_{\perp}}{m c^{2}}\right)^{2}=-\frac{1}{3} r_{e} \gamma^{2} \kappa^{4} r_{\beta}^{2},
$$

where $r_{\beta}$ is the betatron amplitude and we have averaged over the betatron period. For an accelerating beam, the betatron amplitude decreases such that $r_{\beta}=r_{\beta i}\left(\gamma_{i} / \gamma\right)^{1 / 4}$, where $r_{\beta i}$ is the initial betatron amplitude. Assuming the radiation loss is a small perturbation to a constant accelerating gradient $\gamma^{\prime}=d \gamma / d z=k_{p}\left(E_{z} / E_{0}\right) \cos \Psi$, and $\gamma_{f} \gg \gamma_{i}$, the energy loss to synchrotron radiation is

$$
\Delta \gamma \simeq-\frac{2}{15} r_{e} \frac{\kappa^{4}}{\gamma^{\prime}} \gamma_{f}^{5 / 2} r_{\beta i}^{2} \gamma_{i}^{1 / 2}
$$

Averaging over a distribution of particles yields

$$
\langle\Delta \gamma\rangle \simeq-\frac{2}{15} r_{e}\left(\frac{\kappa^{3}}{\gamma^{\prime}}\right) \gamma_{f}^{5 / 2} \epsilon_{n},
$$

where the rms beam size can be expressed in terms of the transverse emittance, $\left\langle r_{\beta i}^{2}\right\rangle=\epsilon_{i} / k_{\beta i}=\epsilon_{n} /\left(\kappa \gamma_{i}^{1 / 2}\right)$ with $\epsilon_{n}=\gamma \epsilon$ the normalized emittance, and the laser-plasma interaction physics is contained in the coefficient $\kappa^{3} / \gamma^{\prime}$. For an LPA in the linear regime $\kappa^{3} / \gamma^{\prime}=\left(8 / k_{p} r_{L}^{3}\right) \times$ $\left(E_{z} / E_{0}\right)^{1 / 2}(\sin \Psi)^{1 / 2} \tan \Psi$. The total power lost to synchrotron radiation is $P_{\text {rad }}=f N m c^{2}\langle\Delta \gamma\rangle$.

On-axis particles will not undergo betatron motion and will not radiate, whereas off-axis particles radiate strongly. The induced rms beam energy spread is given by $\sigma_{\gamma}^{2}=$ $\left\langle(\Delta \gamma-\langle\Delta \gamma\rangle)^{2}\right\rangle$, and using Eq. (B3) the relative energy spread is

$$
\frac{\sigma_{\gamma}}{\gamma_{f}} \simeq \frac{4}{15} r_{e} \epsilon_{n}\left(\frac{\kappa^{3}}{\gamma^{\prime}}\right) \gamma_{f}^{3 / 2} .
$$

For typical collider parameters the induced energy spread from synchrotron radiation is negligible. For example, accelerating in a $n_{0}=10^{17} \mathrm{~cm}^{-3}$ plasma using a resonant laser pulse with $a_{0}=1.5$ and $k_{p} r_{L}=4$, and a beam injected at $\Psi=10^{\circ}$ with $\epsilon_{n}=10^{-7} \mathrm{~m} \mathrm{rad}$, the induced relative rms energy spread is $\sigma_{\gamma} / \gamma_{f} \simeq 10^{-6}$ after acceleration to $E_{b}=0.5 \mathrm{TeV}$.

\section{APPENDIX C: $\gamma \gamma$ COLLIDER}

There are several advantages to considering a gammagamma $(\gamma \gamma)$ collider (or gamma-electron beam collisions) [28], compared to an $e^{+} e^{-}$collider. $\gamma \gamma$ collisions can access many of the lepton interactions available in an $e^{+} e^{-}$collider [29]. In addition, from a collider design viewpoint, a $\gamma \gamma$ collider eliminates the need for a positron beam, and beamstrahlung and beam-beam instabilities are absent.

Photon beams can be generated from the electron beams before the IP via Compton scattering. Consider near backscatter (with small collision angle $\theta \ll 1$ ) of the electron beam with a circularly polarized laser (polarization of the counterpropagating laser opposite that of the electrons). Solving the energy-momentum conservation equations for the electron $\left(u_{\mu}\right)$, laser $\left(k_{L \mu}\right)$, and scattered light $\left(k_{\mu}\right), m c u_{\mu}+\hbar k_{L \mu}=m c u_{\mu}^{\prime}+\hbar k_{\mu}$, yields the photon energy $\hbar \omega=E_{b} x /\left(1+x+a_{L}^{2}\right)$, where $a_{L}^{2}$ is the normalized laser intensity and $x=\left(4 E_{b} \hbar \omega_{L} / m^{2} c^{4}\right) \cos ^{2}(\theta / 2)$. Maximizing the scattered photon energy requires maximizing $x$.

Photons may be lost due to the creation of $e^{+} e^{-}$ pairs (with the associated background issues for the detector). To avoid $e^{+} e^{-}$pair creation requires $\left(\hbar k_{\mu}+\right.$ $\left.\hbar k_{\mu L}\right)^{2}=4 \hbar^{2} k_{L} k \leq\left(2 m_{e} c\right)^{2}\left(1+a_{L}^{2}\right), \quad$ or $\quad x \leq 2(1+$ $\left.a_{L}^{2}\right)(1+\sqrt{2}) \simeq 4.83\left(1+a_{L}^{2}\right)$. For $x=4.8, \hbar \omega \simeq 0.83 E_{b}$ assuming $\quad a_{L} \ll 1, \quad$ and $\hbar \omega_{L} E_{b}=\left(m_{e} c^{2}\right)^{2} x / 4 \simeq$ $0.3(\mathrm{MeV})^{2}$ [28], or

$$
\lambda_{L}[\mu \mathrm{m}] \approx 4 E_{b}[\mathrm{TeV}] .
$$

Equation $(\mathrm{C} 1)$ determines the scattering laser wavelength that maximizes the scattered photon energy while avoiding pair creation. For example, using a solid-state laser with $\hbar \omega_{L}=1.2 \mathrm{eV}$, and scattering off an electron beam with $E_{b}=250 \mathrm{GeV}$, yields photons with energy $\hbar \omega=$ $200 \mathrm{GeV}$.

The luminosity of the photon beams is given by $\mathcal{L}_{\gamma \gamma}=$ $\left(N_{\gamma} / N_{e}\right)^{2} \mathcal{L}_{e^{+} e^{-}}$, where $N_{\gamma}$ is the number of gammas/pulse. Comparable luminosity requires $N_{\gamma} \sim N_{e}$. The cross section for single-photon Compton scattering $(x>1)$ is approximately $\sigma_{\mathrm{C}} \approx \pi r_{e}^{2}(2 \ln x+1) / x$ for $x \gg 1$, where $r_{e}$ is the classical electron radius. For $x=4.8, \sigma_{\mathrm{C}} \approx 2 \times$ $10^{-25} \mathrm{~cm}^{2}$ [29].

For efficient scattering in the linear regime, $2 Z_{R} \approx l_{L}>$ $l_{b}$, with $Z_{R}$ the Rayleigh range, $l_{L}$ the laser pulse length, and $l_{b}$ the electron beam length. To produce $N_{\gamma} \sim N_{e}$ requires $\sigma_{\mathrm{C}} N_{L} / A_{L} \sim 1$, i.e., the thickness of the laser "target" is equal to one Compton scattering length. Here $N_{L}$ is the number of laser photons/pulse and $A_{L} \sim$ $\lambda_{L} Z_{R} / 2=(\pi / 2) r_{L}^{2}$. Setting $\sigma_{C} N_{L} / A_{L}=1$ yields the required laser energy $U_{L}=N_{L} \hbar \omega_{L}=\pi \hbar c Z_{R} / \sigma_{\mathrm{C}}$ or $U_{L}[\mathrm{~J}] \approx 5 Z_{R}[\mathrm{~mm}]$, with $2 Z_{R} \approx l_{L}$. With this laser energy (i.e., one Compton scattering length), the conversion efficiency is $N_{\gamma} / N_{e} \approx 1-e^{-1} \approx 0.65$. Using $U_{L}=\pi \hbar c Z_{R} / \sigma_{\mathrm{C}}$, the normalized intensity can be expressed as $a_{L}^{2} l_{L}=\left(4 r_{e}^{2} / \alpha \sigma_{\mathrm{C}}\right) \lambda_{L}$ or $a_{L}^{2} l_{L}[\mathrm{~mm}] \approx$ $0.9 E_{b}[\mathrm{TeV}]$. The laser energy required is therefore

$$
U_{L}[\mathrm{~J}] \approx\left(2 / a_{L}^{2}\right) E_{b}[\mathrm{TeV}] .
$$

The pulse duration must be long enough such that the intensity is sufficiently low to avoid nonlinear (multiphoton) scattering, i.e., such that $a_{L}^{2}<1$.

In addition, the peak electric field of the laser in the rest frame of the beam must be less than the Schwinger critical field to minimize beamstrahlung. This condition can be expressed as $a_{L}<\lambda_{L} /\left(2 \gamma \lambda_{\mathrm{C}}\right)=2 / x \simeq 0.4$. Setting 
TABLE II. Example parameters for a $0.5 \mathrm{TeV}$ laser-plasma linear $\gamma \gamma$ collider.

\begin{tabular}{lc}
\hline \hline Plasma number density, $n_{0}\left[\mathrm{~cm}^{-3}\right]$ & $10^{17}$ \\
Beam energy, $\gamma m c^{2}[\mathrm{TeV}]$ & 0.25 \\
Geometric luminosity, $\mathcal{L}\left[10^{34} \mathrm{~s}^{-1} \mathrm{~cm}^{-2}\right]$ & 2 \\
Number per bunch, $N\left[10^{9}\right]$ & 4 \\
Collision frequency, $f[\mathrm{kHz}]$ & 15 \\
Number of stages $(1 \mathrm{linac}), N_{\text {stages }}$ & 25 \\
Linac length $\left(1\right.$ beam), $L_{\text {total }}[\mathrm{km}]$ & 0.05 \\
Total wall-plug power, $P_{\text {wall }}[\mathrm{MW}]$ & 80 \\
Compton scattering laser wavelength $[\mu \mathrm{m}]$ & 1 \\
Compton scattering laser energy $[\mathrm{J}]$ & 6 \\
Compton scattering laser duration $[\mathrm{ps}]$ & 7 \\
Compton scattering laser Rayleigh range $[\mathrm{mm}]$ & 1 \\
Compton scattering intensity $\left[10^{18} \mathrm{~W} / \mathrm{cm}^{-2}\right]$ & 0.27 \\
Gamma beam peak energy $[\mathrm{TeV}]$ & 0.2 \\
Conversion efficiency $[e \rightarrow \gamma]$ & 0.65 \\
\hline \hline
\end{tabular}

$a_{L}^{2}=0.1$ yields $l_{L}[\mathrm{~mm}] \approx 9 E_{b}[\mathrm{TeV}]$, and $U_{L}[\mathrm{~J}] \approx$ $23 E_{b}[\mathrm{TeV}]$.

For example, a beam with $E_{b}=250 \mathrm{GeV}\left(E_{\mathrm{cm}}=\right.$ $0.5 \mathrm{TeV}$ ) requires a $1 \mu \mathrm{m}$ wavelength, $6 \mathrm{~J}, 7 \mathrm{ps}$ laser, with $Z_{R}=1 \mathrm{~mm}$ and $I=2.7 \times 10^{17} \mathrm{~W} / \mathrm{cm}^{2}$. The gamma-ray energy peaks at $0.8 E_{b}=200 \mathrm{GeV}$, with luminosity $\mathcal{L}_{\gamma \gamma} / \mathcal{L}_{e^{+} e^{-}} \approx\left(N_{\gamma} / N_{e}\right)^{2} \approx 0.4$. Note that, although the interaction of the laser with the electron beam is at a point where the electron beam cross section is approximately that of the laser, the scattered light is along the direction of the electron beam (since $E_{b} \gg \hbar \omega_{L}$ ) and will converge at the IP. The interaction must be done sufficiently close to the IP such that the natural spreading of the gamma rays, with divergence $\left(1+x+a_{L}^{2}\right)^{1 / 2} m c^{2} / E_{b}$, does not significantly reduce the collisions. Table II shows a $0.5 \mathrm{TeV} \gamma \gamma$ collider example based on $n=10^{17} \mathrm{~cm}^{-3}$ LPA stages (i.e., the LPA and collider parameters of Table I producing $250 \mathrm{GeV}$ electron beams).

[1] J. Ellis and I. Wilson, Nature (London) 409, 431 (2001).

[2] I. Hinchliffe and M. Battaglia, Phys. Today 57, No. 9, 49 (2004).

[3] G. Dugan, in Advanced Accelerator Concepts, edited by V. Yakimenko (AIP, New York, 2004), Vol. 737, pp. 29-60.

[4] E. Esarey, C. B. Schroeder, and W. P. Leemans, Rev. Mod. Phys. 81, 1229 (2009).

[5] S. P. D. Mangles et al., Nature (London) 431, 535 (2004); C. G. R. Geddes et al., ibid. 431, 538 (2004); J. Faure et al., ibid. 431, 541 (2004).

[6] W. Leemans and E. Esarey, Phys. Today 62, No. 3, 44 (2009).

[7] C. B. Schroeder, E. Esarey, C. G. R. Geddes, C. Tóth, and W. P. Leemans, in Advanced Accelerator Concepts, edited by C. B. Schroeder, E. Esarey, and W. Leemans (AIP, New York, 2009), Vol. 1086, pp. 208-214.
[8] W. P. Leemans, B. Nagler, A. J. Gonsalves, C. Tóth, K. Nakamura, C. G. R. Geddes, E. Esarey, C. B. Schroeder, and S. M. Hooker, Nature Phys. 2, 696 (2006).

[9] K. Nakamura, B. Nagler, C. Tóth, C. G. R. Geddes, C. B. Schroeder, E. Esarey, W. P. Leemans, A. J. Gonsalves, and S. M. Hooker, Phys. Plasmas 14, 056708 (2007).

[10] A. Pukhov and J. Meyer-ter-Vehn, Appl. Phys. B 74, 355 (2002).

[11] W. Lu, M. Tzoufras, C. Joshi, F. S. Tsung, W. B. Mori, J. Vieira, R. A. Fonseca, and L. O. Silva, Phys. Rev. ST Accel. Beams 10, 061301 (2007).

[12] N.E. Andreev, L. M. Gorbunov, V.I. Kirsanov, K. Nakajima, and A. Ogata, Phys. Plasmas 4, 1145 (1997).

[13] K. V. Lotov, Phys. Plasmas 14, 023101 (2007).

[14] X. Wang, P. Muggli, T. Katsouleas, C. Joshi, W. B. Mori, R. Ischebeck, and M.J. Hogan, Phys. Rev. ST Accel. Beams 12, 051303 (2009).

[15] E. Cormier-Michel, E. Esarey, C. G. R. Geddes, C. B. Schroeder, W. P. Leemans, D. L. Bruhwiler, B. Cowan, and K. Paul, in Proceedings of the 10th International Computational Accelerator Physics Conference (JACoW, Geneva, 2009) [http://www.jacow.org].

[16] M. Reiser, Theory and Design of Charged Particle Beams (Wiley-VCH, Weinheim, 2008), 2nd ed.

[17] J. B. Rosenzweig, A. M. Cook, A. Scott, M. C. Thompson, and R. B. Yoder, Phys. Rev. Lett. 95, 195002 (2005).

[18] T. Katsouleas, S. Wilks, P. Chen, J. M. Dawson, and J. J. Su, Part. Accel. 22, 81 (1987).

[19] M. Tzoufras, W. Lu, F. S. Tsung, C. Huang, W. B. Mori, T. Katsouleas, J. Vieira, R. A. Fonseca, and L. O. Silva, Phys. Rev. Lett. 101, 145002 (2008).

[20] C. G. R. Geddes, C. Toth, J. van Tilborg, E. Esarey, C. B. Schroeder, J. Cary, and W. P. Leemans, Phys. Rev. Lett. 95, 145002 (2005).

[21] P. Sprangle, B. Hafizi, J. R. Peñano, R. F. Hubbard, A. Ting, C. I. Moore, D. F. Gordon, A. Zigler, D. Kaganovich, and T. M. Antonsen, Jr., Phys. Rev. E 63, 056405 (2001).

[22] W. Rittershofer, C. B. Schroeder, E. Esarey, F. J. Grüner, and W. P. Leemans, Phys. Plasmas 17, 063104 (2010).

[23] B. A. Shadwick, C. B. Schroeder, and E. Esarey, Phys. Plasmas 16, 056704 (2009).

[24] B. Dromey, S. Kar, M. Zepf, and P. Foster, Rev. Sci. Instrum. 75, 645 (2004).

[25] C. Thaury et al., Nature Phys. 3, 424 (2007).

[26] D. Panasenko, A. J. Shu, A. Gonsalves, K. Nakamura, N. H. Matlis, C. Toth, and W. P. Leemans, J. Appl. Phys. 108, 044913 (2010).

[27] B. A. Shadwick, G. M. Tarkenton, E. H. Esarey, and W. P. Leemans, IEEE Trans. Plasma Sci. 30, 38 (2002).

[28] V. Telnov, Nucl. Instrum. Methods Phys. Res., Sect. A 355, 3 (1995).

[29] B. Badelek et al., Int. J. Mod. Phys. A 19, 5097 (2004).

[30] Handbook of Accelerator Physics And Engineering, edited by A.W. Chao and M. Tigner (World Scientific, Singapore, 1999), 3rd printing.

[31] E. Esarey, R. F. Hubbard, W. P. Leemans, A. Ting, and P. Sprangle, Phys. Rev. Lett. 79, 2682 (1997).

[32] C. B. Schroeder, P. B. Lee, J. S. Wurtele, E. Esarey, and W. P. Leemans, Phys. Rev. E 59, 6037 (1999).

[33] S. Bulanov, N. Naumova, F. Pegoraro, and J. Sakai, Phys. Rev. E 58, R5257 (1998). 
[34] P. Tomassini, M. Galimberti, A. Giulietti, D. Giulietti, L. A. Gizzi, L. Labate, and F. Pegoraro, Phys. Rev. ST Accel. Beams 6, 121301 (2003).

[35] J. Faure, C. Rechatin, A. Norlin, A. Lifschitz, Y. Glinec, and V. Malka, Nature (London) 444, 737 (2006).

[36] C. G. R. Geddes, K. Nakamura, G. R. Plateau, Cs. Tóth, E. Cormier-Michel, E. Esarey, C. B. Schroeder, J. R. Cary, and W. P. Leemans, Phys. Rev. Lett. 100, 215004 (2008).

[37] G. Dugan (private communication).

[38] D. Umstadter, J. K. Kim, and E. Dodd, Phys. Rev. Lett. 76, 2073 (1996).

[39] D. Umstadter, E. Esarey, and J. Kim, Phys. Rev. Lett. 72, 1224 (1994).
[40] J. R. Cary, R. E. Giacone, C. Nieter, and D. L. Bruhwiler, Phys. Plasmas 12, 056704 (2005).

[41] A. Ikesue and Y. L. Aung, Nat. Photon. 2, 721 (2008).

[42] A. Butler, D. J. Spence, and S. M. Hooker, Phys. Rev. Lett. 89, 185003 (2002).

[43] N. Kirby, M. Berry, I. Blumenfeld, M. J. Hogan, R. Ischebeck, and R. Siemann, in Proceedings of PAC07 (JACoW, Geneva, 2007), pp. 3097-3099 [http://www .jacow.org].

[44] D. R. Nicholson, Introduction to Plasma Theory (Krieger, Malabar, FL, 1992).

[45] J.D. Jackson, Classical Electrodynamics (Wiley, New York, 1975), 2nd ed. 